\title{
Salud, enfermedad y muerte en la población manteña de Japoto: las evidencias osteológicas y dentales
}

Santé, maladie et mort chez la population manteña de Japoto : les preuves ostéologiques et dentaires

Health, disease and death from Manteño population in Japoto: osteologiocal and dental evidence

\section{Tania Delabarde}

\section{(2) OpenEdition}

\section{Journals}

Edición electrónica

URL: http://journals.openedition.org/bifea/1742

DOI: $10.4000 /$ bifea. 1742

ISSN: 2076-5827

Editor

Institut Français d'Études Andines

Edición impresa

Fecha de publicación: 1 diciembre 2010

Paginación: 531-550

ISSN: 0303-7495

Referencia electrónica

Tania Delabarde, «Salud, enfermedad y muerte en la población manteña de Japoto: las evidencias osteológicas y dentales », Bulletin de l'Institut français d'études andines [En línea], 39 (3) | 2010,

Publicado el 01 junio 2011, consultado el 09 diciembre 2020. URL : http://journals.openedition.org/ bifea/1742 ; DOI : https://doi.org/10.4000/bifea.1742

\section{cc)}

Les contenus du Bulletin de l'Institut français d'études andines sont mis à disposition selon les termes de la licence Creative Commons Attribution - Pas d'Utilisation Commerciale - Pas de Modification 4.0 International. 


\title{
Salud, enfermedad y muerte en la población manteña de Japoto: las evidencias osteológicas y dentales
}

\author{
Tania Delabarde*
}

\section{Resumen}

En los últimos años, el avance de las investigaciones sobre la biología de las poblaciones arqueológicas ha sido fundamental debido al aporte de la genética y de los análisis bioquímicos, que llevaron a un mejor conocimiento del origen de los poblamientos, el diagnóstico preciso de algunas enfermedades y los modos de vida con el estudio de la dieta. Desde 2004, las investigaciones hechas en el sitio de Japoto (el Ecuador, provincia de Manabí) permitieron estudiar a la población manteña, desde el punto de vista cultural y biológico, con el estudio de sus prácticas funerarias, la determinación de lesiones traumáticas e infecciosas, el análisis de elementos traza y la comparación con vestigios materiales para evaluar las conductas alimentarias. Los resultados de este estudio bioantropológico completan los conocimientos sobre la vida y muerte de las poblaciones prehispánicas, lejos de la imagen idílica de un Nuevo Mundo sin enfermedad. La población manteña de Japoto supo dominar su medio ambiente, adaptándose a las condiciones climáticas y geográficas, y aprovechando los recursos naturales para su alimentación y curar las enfermedades.

Palabras clave: antropobiología, cultura manteña, Ecuador

\section{Santé, maladie et mort chez la population manteña de Japoto : les preuves ostéologiques et dentaires}

\section{Résumé}

Ces dernières années, les progrès dans la recherche sur la biologie des populations archéologiques ont été significatifs grâce à la génétique et aux analyses biochimiques. Celles-ci ont permis une

* Institut de Médecine Légale. Universidad de Strasbourg, 11 rue Humann, 67085 Strasbourg-Cedex, Francia. E-mail: tdelabarde@hotmail.com 
meilleure compréhension de l'origine des populations, et de leurs modes de vie grâce à l'étude des comportements alimentaires et un diagnostic précis de certaines maladies. Depuis 2004, les fouilles archéologiques du site de Japoto (Équateur, Province de Manabí) ont permis d'étudier la population manteña, dans une perspective culturelle et biologique. Ont été étudiées les pratiques funéraires, la détermination des lésions traumatiques et infectieuses, I'analyse des éléments traces et la comparaison avec les vestiges archéologiques pour évaluer les comportements alimentaires. Les résultats de cette étude bio-anthropologique s'ajoutent au corpus des données sur la vie et la mort des populations préhispaniques, loin de l'image idyllique d'un Nouveau Monde exempt de maladie. La population manteña de Japoto a su tirer profit de son environnement en s'adaptant aux contraintes climatiques et géographiques de son milieu, y puisant les ressources nécessaires pour s'alimenter et lutter contre les maladies.

Mots clés : anthropologie biologique, culture manteña, Équateur

\title{
Health, disease and death from Manteño population in Japoto: osteologiocal and dental evidence
}

\begin{abstract}
In recent years, significant advances in research have been made on the biology of archaeological populations with genetic and biochemical analysis providing a better understanding of the origin of populations, lifestyles and diet, and accurate diagnosis of certain diseases. Since 2004, archaeological excavations on the site Japoto (Ecuador, Manabí Province) allowed the analysis of the Manteño population from a cultural and biological perspective, with the study of burial practices, identification of injuries and infectious diseases, trace element analysis and comparison with the archaeological material to assess eating behaviors. Far from the idyllic image of a New World free from disease before the Spanish conquest, the results of this study add to the bio-anthropological corpus of data on the life and death of Pre-Hispanic populations. The Manteños from Japoto took advantage of the site's environment, adapting to climatic and geographical constraints, and taking benefit of natural resources for food and treatment to fight against diseases.
\end{abstract}

Key words: bioanthropology, Manteño culture, Ecuador

\section{INTRODUCCIÓN}

El estudio de los restos humanos por de la antropología física ha sido siempre una de las fuentes más importantes para el conocimiento biológico y cultural de las poblaciones arqueológicas. En los últimos veinte años, el desarrollo de la arqueometría, ciencia que emplea métodos físicos o químicos para los estudios arqueológicos, permitió gracias sus avances tecnológicos, obtener datos inéditos en los campos de la genética (filiación, origen de las poblaciones) y de la biología humana (paleopatología, alimentación) a través de los análisis del ADN antiguo, de isótopos estables y elementos trazas.

La paleopatología, el estudio de las enfermedades de las poblaciones antiguas, se determina en base a los estudios de restos humanos (momias, huesos, dientes, coprolitos), las informaciones históricas y la representación de las enfermedades 
en los soportes materiales (Wells, 1965). Otros investigadores se concentran más en la descripción de las enfermedades evidenciadas en los tejidos óseo y dental con un enfoque hacia el origen, la distribución y características de las mismas (Steckel \& Rose, 2002; Ortner, 2003). Esta disciplina también ha desarrollado su campo de investigación y sus resultados, lo que permite hoy en día diagnosticar las enfermedades no solo mediante la descripción y caracterización de las lesiones, sino con la identificación del agente patógeno gracias a los análisis genéticos.

En la América Latina, el examen de numerosas momias con estudios radiográficos, endoscópicos e histopatológicos ha incrementando de manera notable los conocimientos sobre el estado sanitario de las poblaciones precolombinas. En el sitio de Japoto (Ecuador) el estudio antropológico de los restos humanos iniciado desde el año 2004 permite presentar un panorama de la vida y muerte de una población precolombina en el periodo de Integración, mediante el aporte de los análisis bioquímicos (ADN e isótopos estables).

\section{SALUD Y ENFERMEDADES EN EL MUNDO PREHISPÁNICO}

Ayer como hoy, las epidemias tuvieron una influencia importante en la evolución biológica y cultural de las poblaciones. Una revisión de la bibliografía sobre este tema da cuenta de los avances de las investigaciones desde la imagen idílica de un nuevo mundo precolonial sin epidemias hacia un perfil de vida y muerte parecido al viejo mundo en varios aspectos. Durante mucho tiempo, los investigadores afirmaron que el factor del aislamiento biológico en el nuevo continente desde el Pleistoceno hasta la llegada de los españoles preservó las poblaciones precolombinas de los grandes focos de enfermedades infecciosas que se desarrollaron en otros continentes. Basándose en fuentes históricas de la conquista española, Diomedi (2003: 21-22) argumentó incluso la existencia de una guerra biológica con utilización de agentes infecciosos durante la época de la Conquista en el nuevo continente: los capitanes de Francisco Pizarro advertieron la importante mortalidad producida por la viruela en las poblaciones indígenas, y a raíz de esto, solían mandar por delante de sus tropas a soldados o esclavos portando lanzas contaminadas con secreciones obtenidas de enfermos de la viruela. Cuando las tropas abandonaban los campamentos, dejaban las prendas contaminadas de los enfermos de viruela o las regalaban a las poblaciones indígenas.

La Conquista estuvo sin duda marcada por el impacto de las patologías infecciosas y las transmisiones de enfermedades foráneas para las cuales las poblaciones precolombinas no poseían defensas. Algunos autores hablan de colapso demográfico con la llegada de los colonizadores europeos y de los esclavos africanos más tarde. Mientras que las poblaciones originarias del Viejo Mundo crecían rápidamente después de la Conquista, las sociedades nativas sufrieron no solo de las enfermedades infecciosas sino también de la servidumbre, explotación y muerte por parte de los colonizadores. Las estimaciones sobre la cantidad de habitantes en América a la llegada de los españoles varían mucho entre los investigadores, sin embargo existe un consenso general sobre el hecho de que la 
población americana disminuyó de manera considerable en el primer siglo de la Conquista (Denevan et al., 1973).

Las crónicas españolas constituyeron la primera fuente de datos sobre la salud y las enfermedades de las poblaciones precolombinas pero la mayoría de los textos históricos de escritores europeos o nativos reportaron una imagen idílica de la vida y salud de los tiempos precoloniales. En cambio los relatos del padre Pablo Joseph de Arriaga, de Felipe Guamán Poma de Ayala y Garcilaso de la Vega en el Perú registraron abundante información sobre la salud y las enfermedades en la sociedad inca. En los últimos 30 años, un número creciente de investigadores (Austin Alchon, 1999: 201) empezaron a poner en duda la imagen idílica del Nuevo Mundo y gracias a los avances en paleopatología y paleodemografía, se pudo demonstrar la existencia de epidemias, hambrunas y guerras, factores de reducción de las expectativas de vida y el aumento de la tasa de mortalidad de las poblaciones precolombinas.

Hoy en día, gracias a los avances de los análisis químicos y bioquímicos se ha podido establecer que los patrones de enfermedad del Nuevo y del Viejo mundo tienen más similitudes que diferencias. La mayor diferencia en el pasado consiste en la ausencia en América de varias enfermedades masivas específicas como la viruela, el sarampión y la peste bubónica.

Dentro del estudio de los patrones de enfermedad se deben considerar los factores económicos y sociales, los recursos del medio ambiente, la densidad de población y los tipos de sociedad (cazadores, recolectores, agricultores). El sedentarismo y la concentración de población son dos factores importantes que favorecen la proliferación de enfermedades infecciosas. Las infecciones gastrointestinales por contaminación del agua se han multiplicado con la sedentarización.

La dieta influyía directamente sobre la nutrición y el estado sanitario de las poblaciones afectando su desarrollo. Los cazadores-recolectores tenían una dieta más variada que las sociedades sedentarias cuya dieta se limitaba a dos o tres cultivos, entre ellos el maíz, deficiente en varios elementos nutritivos importantes. Los estudios osteológicos demostraron un patrón de desnutrición y una disminución de la estatura consecuencia del deterioro de la salud en las sociedades sedentarias y agrícolas del Nuevo Mundo (Storey, 1992: 231). El estrés laboral, por ejemplo, era importante en las poblaciones precolombinas por la escasez de animales de carga, a excepción de los camélidos en los Andes Centrales. Por otro lado, no existían muchas especies de cuadrúpedos y las poblaciones precolombinas domesticaron menos especies animales, vehículos de muchos agentes patógenos como la viruela y el sarampión en el viejo continente.

Los diferentes estudios sobre las poblaciones precolombinas demuestran una mortalidad infantil alta y una esperanza de vida baja. Las expectativas de vida en las sociedades precolombinas permanecen bajas en comparación con el nuevo continente (Austin Alchon, 1999: 210). Ubelaker (2003: 270) menciona por ejemplo una esperanza de vida de 24 años al nacer en el Ecuador por el Periodo Formativo, ligeramente reducida sin embargo en el Periodo Precerámico y en los periodos Precontactos y subiendo en los periodos Históricos. 
Las enfermedades letales más frecuentes eran infecciones respiratorias y gastrointestinales severas. Los estudios osteológicos demuestraron que al menos el tifus (endémico y epidémico) y la influenza fueron las dos enfermedades epidémicas presentes tanto en el viejo como en el nuevo continente. Las infecciones respiratorias agudas como la neumonía eran una causa de muerte frecuente en las sociedades sedentarias. Se encontraron evidencias osteológicas de tuberculosis en varios sitios arqueológicos con fechas tempranas y tardías demostrando la antigüedad y permanencia de esta enfermedad (Buikstra, 1992: 311; Lombardi, 2000: 57; Ortner, 2003: 227) en el nuevo continente. Las complicaciones posparto eran la principal causa de muerte entre las mujeres (Buikstra, 1992: 305). Los hombres sufrían más frecuentemente de lesiones traumáticas por violencia social o accidentes que las mujeres (Jaffe, 1992: 60; Ubelaker, 2003: 271). El cronista español Pedro Cieza de León (1984 [1553]: 224) da un ejemplo de la violencia del ejército inca al relatar la masacre de 20000 hombres de la región de Otavalo cerca de la laguna de Yaguarcocha en 1500.

La paleopatología en la América del Sur se desarrolló en sus inicios gracias a las colectas de material óseo por parte de los exploradores. Las primeras investigaciones se enfocaron en la descripción de las lesiones del tejido óseo para evolucionar luego hacia una perspectiva poblacional del análisis de las enfermedades y, en los últimos años, hacia la adaptabilidad humana a través de los estudios de paleodieta y paleoepidemiología (Verano \& Lombardi, 1999). Las técnicas moleculares permiten hoy en día un diagnóstico preciso de las enfermedades que provocan lesiones del tejido óseo. Los cambios patológicos en el tejido óseo resultan del desequilibrio entre las medidas normales de resorción y formación ósea y en los desordenes del desarrollo (White, 2000: 381).

\section{HUESOS Y DIENTES: TESTIGOS BIOLÓGICOS Y CULTURALES DE LA POBLACIÓN MANTEÑA DE JAPOTO}

El tejido óseo es un tejido conectivo, constituyente principal de los huesos en los vertebrados. La matriz ósea compuesta de células y componentes extracelulares calcificados se caracteriza por su rigidez y su gran resistencia, explicando la conservación de restos óseos muy antiguos. Gracias a la actividad de las células el tejido óseo se renueva constantemente y permite evidenciar los episodios de estrés alimentario, infeccioso o traumatológico que suelen ocurrir. Todos estos datos reflejan el estado sanitario de las poblaciones arqueológicas.

El número total de enfermedades que afectan el esqueleto es relativamente reducido porque la mayoría de las que son fatales son de breve duración y afectan principalmente los tejidos blandos. Las enfermedades que afectan principalmente el esqueleto humano son infecciones crónicas bacteriales y lesiones degenerativas como la artritis reumática (Ortner, 2003).

Las lesiones infecciosas en los restos óseos secos pueden ser examinadas macroscópicamente y luego confirmadas gracias a los cortes histológicos, toma de radiografías $\mathrm{X}$, tomodensitometría (técnica de medición del contenido en materia 
mineral de un tejido, basada en su capacidad de atenuación de los fotones de los rayos $X$, estudiada mediante tomografía computarizada) y análisis genéticos para determinar precisamente el germen patogénico.

Sin embargo el estado de conservación de los huesos varía mucho de un sitio arqueológico a otro. El tipo de suelo y sus características físico químicas, las condiciones ambientales y el tiempo que transcurrió desde el entierro de un individuo son los factores principales de la degradación o preservación de los huesos. El grano de la roca y la compactación de los sedimentos influyen también en la degradación de los restos óseos así como las raíces y los demás factores tafonómicos.

\section{1. Estudio bioantropológico de los restos humanos en Japoto}

En el sitio de Japoto (ver Bouchard en este mismo volumen), el clima tropical, la alternancia de episodios de sequedad y lluvia intensa (fenómeno del Niño) junto a la acidez del subsuelo fragilizaron el material arqueológico a lo largo de los años. Los suelos ácidos, con pH inferior a 7 afectaron la fracción mineral de los huesos y la humedad de los suelos creó microorganismos que destruyeron poco a poco los restos óseos.

Pese a estas condiciones, un número importante de datos biológicos fueron registrados a partir de los restos humanos encontrados en el sitio de Japoto. La mayoría del material óseo proviene de estructuras funerarias (Delabarde, 2006; 2010).

Desde 2004, fueron excavado un total de 32 depósitos funerarios ubicados en las diferentes tolas de Japoto. La mayoría de los depósitos funerarios fueron encontrados en la tola $\mathrm{J} 7(\mathrm{~N}=10 ; 7$ son secundarios y 3 primarios). El Número Mínimo de Individuos no incluyó los huesos humanos aislados encontrados en el sitio en general.

El número total de individuos recuperados en depósitos funerarios en Japoto (tolas J5, J6 y J7) es de 39, con 27 adultos y 12 subadultos (cuadro 1). Este número mínimo de individuos (NMI), sin duda, puede subestimar el número real de individuos originalmente en el sitio debido a prácticas funerarias donde prevalecen los depósitos secundarios y la posible selección de los individuos. No hemos considerados los restos humanos aislados encontrados en las demás tolas para el cálculo del NMI. De los 27 adultos, solo se ha podido determinar el sexo para ocho individuo (6 varones y 2 mujeres), sobre la base del dimorfismo sexual de la cintura pélvica y del cráneo, pero no se ha podido determinar el sexo de 19 individuos debido a problemas de conservación y prácticas funerarias. Los métodos utilizados en la evaluación de la edad para subadultos incluyen el desarrollo óseo y dental (Scheuer \& Black, 2000) y, para los adultos, los procesos degenerativos al nivel de la sínfisis púbica y de la cuarta costilla (Brooks \& Suchey, 1990; Iscan et al., 1984; 1985). Los individuos que no han podido ser sexuados son designados como adultos. La estatura no se ha podido estimar para la mayoría de los individuos, debido al alto nivel de fragmentación de huesos largos o a su ausencia (un solo individuo está representado por un número 
Japoto, Ecuador: salud, enfermedad y muerte. Evidencias osteológicas y dentales

Cuadro 1 - Resultados del análisis antropológico de los restos humanos en Japoto (tolas J5, J6 y J7) (*muestra de isotopos estables)

\begin{tabular}{|c|c|c|c|c|}
\hline $\begin{array}{l}\text { Referencia } \\
\text { estructura } \\
\text { funeraria }\end{array}$ & Edad & Sexo & $\begin{array}{l}\text { Estatura } \\
(\mathbf{c m})\end{array}$ & Lesiones patológicas \\
\hline $\mathrm{J} 7-\mathrm{ZO}^{*}$ & 1adulto & I & & \\
\hline $\mathrm{J7-ZOB}$ & $1(30-40)$ & $M$ & $159,03 \pm 3,41$ & \\
\hline $\mathrm{J} 7-\mathrm{Z1}{ }^{*}$ & 3 adultos & $\begin{array}{l}2 \mathrm{M} \\
1 \mathrm{~F}\end{array}$ & & \\
\hline $\mathrm{J} 7-\mathrm{Z} 2 *$ & $\begin{array}{l}2 \text { adultos } \\
1 \text { niño }\end{array}$ & 1 & $153,34 \pm 3,41$ & $\begin{array}{l}\text { Periostitis moderada no es- } \\
\text { pecífica/ligera deformación } \\
\text { en la superficie del hueso } \\
\text { peroné }\end{array}$ \\
\hline $\mathrm{J} 7-\mathrm{Z3}$ & $\begin{array}{l}5 \text { adultos } \\
2 \text { niños }\end{array}$ & I & & $\begin{array}{l}\text { Fractura desplazada con cal } \\
\text { óseo en el peroné izquierdo } \\
\text { (ind\#2) }\end{array}$ \\
\hline J7-E1 & $\begin{array}{c}1 \\
(21 \\
\text { meses } \pm 3) \\
\end{array}$ & I & & \\
\hline J7-E2 & $1(30-40)$ & M & & $\begin{array}{c}\text { osteofitosis moderada en la } \\
\text { columna lumbar, moderado } \\
\text { cambios artríticos en las } \\
\text { facetas articulares }\end{array}$ \\
\hline J7-E3 & 1 adulto & I & & \\
\hline J7-PW1 & $\begin{array}{c}1 \text { niño } \\
(9 \\
\text { meses } \pm 3)\end{array}$ & I & & $\begin{array}{l}\text { hiperostosis porótica en diá- } \\
\text { fisis del húmero izquierdo } \\
\text { y en ambos techos orbi- } \\
\text { tales con lesiones de cribra } \\
\text { orbitalia }\end{array}$ \\
\hline J7-PW2 & $\begin{array}{c}1 \text { niño } \\
(3 \\
\text { meses } \pm 3)\end{array}$ & 1 & & \\
\hline $\begin{array}{c}\text { J5-R8-R1-TAN } \\
\text { J5-R18 }\end{array}$ & $\begin{array}{c}5 \text { adultos } \\
2 \text { niños } \\
(3 \text { meses } \pm 3) \\
1 \text { niño }\end{array}$ & $\begin{array}{l}1 \\
1\end{array}$ & & \\
\hline
\end{tabular}




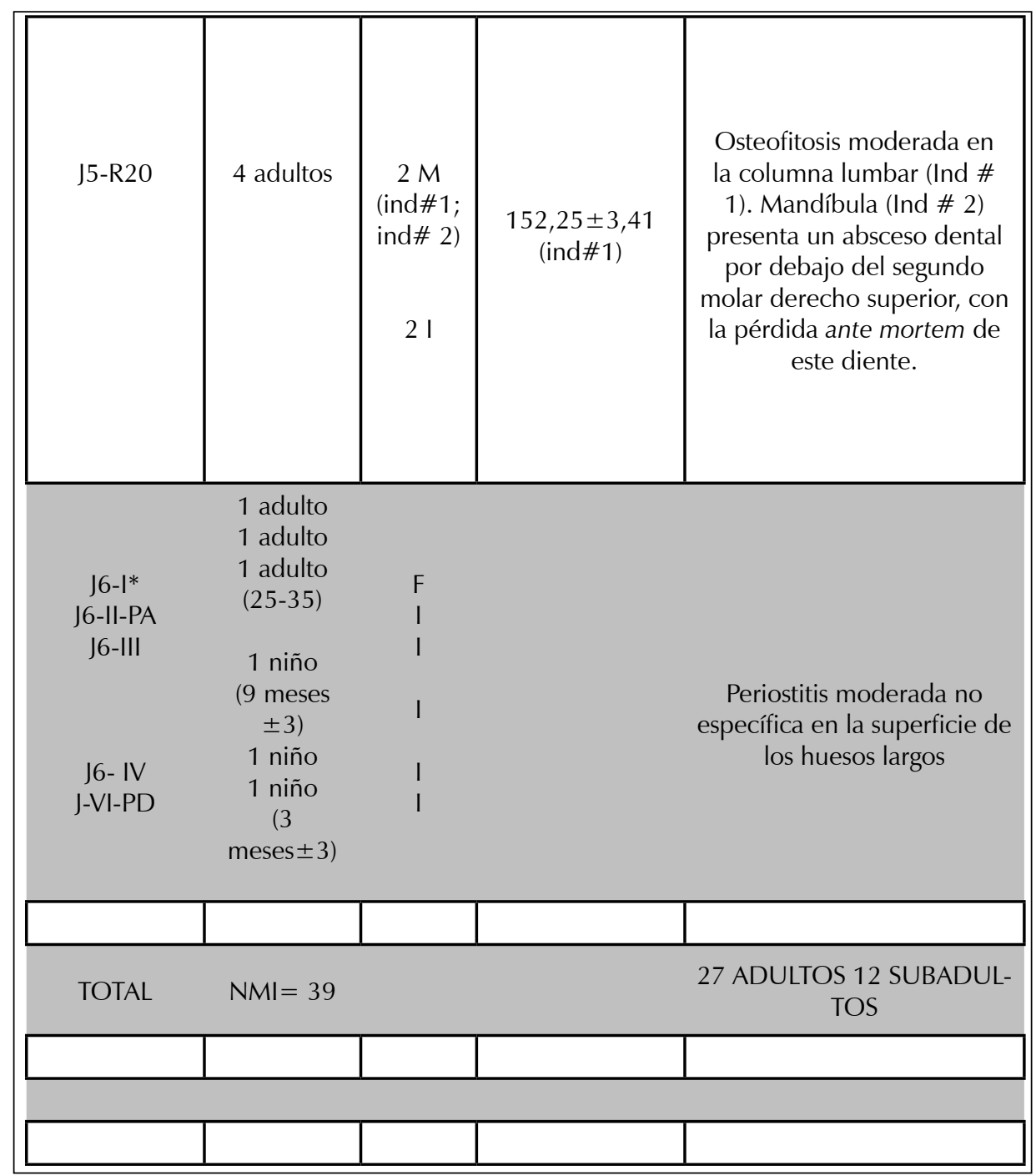

limitado de huesos en los depósitos secundarios). Las estimaciones de la estatura en vida solo están disponibles para 3 individuos (3 varones) con la longitud del fémur, utilizando las ecuaciones de Genovés (1967: 76). La elección de este método en lugar de las basadas en otros elementos esqueléticos se correlaciona con la posibilidad de hacer comparaciones con otros estudios antropológicos. Los resultados presentados en el cuadro 1 son consistentes con los datos biológicos contemporáneos recogidos por antropólogos físicos en la costa ecuatoriana (Ubelaker, 1981; 1997; 2003; Graber \& Jastremski, 2009).

A pesar del estado de conservación de los restos humanos y las prácticas funerarias, fueron registradas evidencias patológicas en siete individuos, cuatro adultos y un 
niño de la tola $\mathbf{J 7}$ y un adulto de tola J6. El cráneo de un niño de 9 meses $( \pm 3$ meses) presenta evidencia de dos lesiones poróticas localizadas al nivel de los techos orbitales y de los arcos supraorbitales (fig. 1). Otra lesión porótica también se registró en la porción distal de la diáfisis del húmero izquierdo. El término hiperostosis porótica se refiere a una expansión del hueso esponjoso en el diploe de la bóveda craneal (por lo general los parietales), así como en los techos órbitales (cribra orbitalia). La etiología no está clara, y aunque tradicionalmente se cree que representa una respuesta ósea a la anemia, o a enfermedades neoplásicas y metabólicas, también puede ser un indicador general de salud pobre o de una dieta desiquilibrada. Según Ubelaker, la fuente de la anemia en poblaciones prehispánicas sería la dieta o problemas de morbilidad. El culpable en la dieta por lo general es el maíz, porque el maíz es bajo en hierro y contiene fitatos que pueden inhibir la absorción del hierro. El resultado es la anemia por deficiencia de hierro, que a su vez desencadena la respuesta ósea (Ubelaker, 1997: 40). Sin embargo, investigaciones recientes tienden a demostrar que la frecuencia de cribra orbitalia en las poblaciones antiguas no necesariamente reflejan la frecuencia de anemia (Walper et al., 2004). Los estudios hematológicos clínicos modernos subrayan más la importancia de la deficiencia de vitamina B12 por la dieta materna que carece de alimentos de origen animal en los lactantes (Walker et al., 2009).

El esqueleto casi completo recuperado en el área de PX (E2) de la tola J7 representa un hombre entre 30-40 años de edad. La columna lumbar muestra osteofitosis moderada con la formación de espículas y picos artríticos en las superficies articulares (fig. 2). Moderados cambios artríticos se observan también en la epífisis

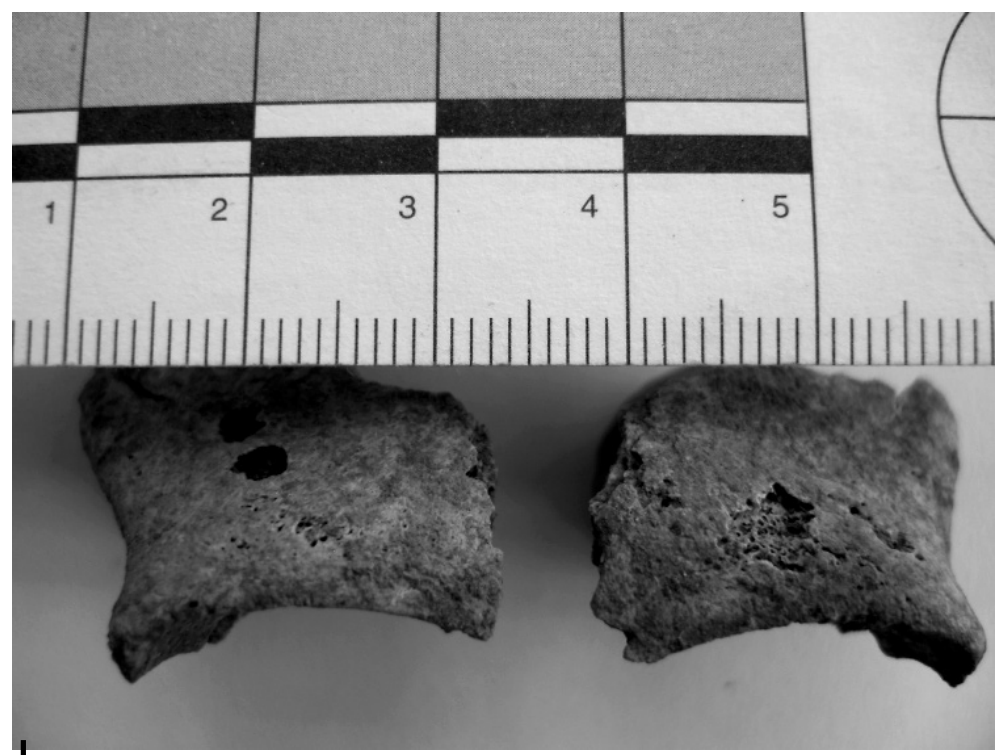

Figura 1 - Dos lesiones poróticas localizadas al nivel de los techos orbitales y los arcos supraorbitales del cráneo de un niño de 9 meses ( \pm 3 meses) (Tola J7, PW1) 


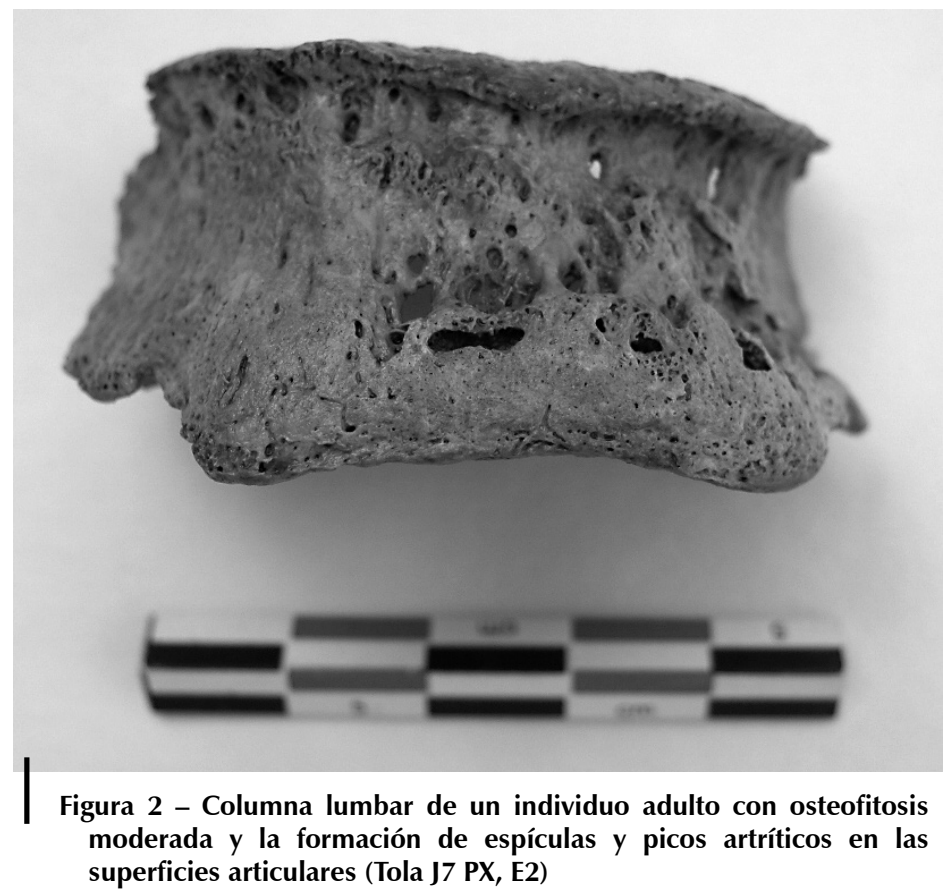

distal de ambos húmeros a nivel de la fosa del olecranon, y en las fases articulares de la epífisis distal de los dos primeros metacarpianos. También se observa osteofitosis en la columna lumbar del esqueleto del depósito principal R20 de la tola J5. Los osteofitos son excrecencias óseas que crecen a partir de los límites del hueso normal, como consecuencia del envejecimiento, de la degeneración ósea producida por enfermedades reumáticas y también actividades específicas como el transporte de carga pesada.

El 1/3 distal de peroné izquierdo de un adulto se ha recuperado en el depósito secundario Z2 de la tola $\mathrm{J}$, mostrando lesiones moderadas de periostitis con pequeñas espículas de hueso que se encuentran perpendiculares al hueso cortical subyacente, asociadas a una ligera deformación de la superficie del hueso. Un corte de la lesión ósea muestra bajo microscopio que la superficie es irregular y porosa indicando una inflamación activa en el momento de la muerte. Un individuo adulto recuperado de un depósito secundario en una urna funeraria (J6-III) en la tola J6 también presenta lesiones de periostitis en la superficie de todos los huesos largos (fig. 3). Las lesiones del periostio se encuentran entre las anomalías más comunes en los esqueletos arqueológicos, y se caracterizan por los cambios patológicos del hueso subyacente. Según Ortner, la periostitis es el resultado de dos condiciones patológicas, traumas e infecciones, y tiene múltiples posibilidades de diagnóstico, tales como las úlceras crónicas de la piel y la sífilis (Ortner, 2003). Los datos dentales en Japoto son limitados debido a la condición de los restos y el bajo porcentaje de las piezas dentales encontrados 
en los depósitos funerarios. Los dientes recuperados muestran la presencia de un cálculo dental moderado. Sólo se registró un ejemplo de enfermedad dental. La mandíbula del adulto (ind\#2) que se ha encontrado en un depósito secundario de la tola J5 presenta un absceso dental por debajo del segundo molar superior derecho, con la pérdida ante mortem de este diente (fig. 4). Según otros estudios antropológicos, las tasas más pequeñas de abscesos dentales y pérdida dental ante mortem se encuentran a fines del Periodo Precontacto y en el Periodo Histórico Temprano (Ubelaker, 1981; 1997; 2003).

En el Ecuador, Ubelaker estudió un número total de 1637 esqueletos provenientes de 23 sitios arqueológicos con diferentes fechados entre el Precerámico y el Periodo de Integración y esqueletos del Convento de San Francisco en Quito fechados en los siglos XIX y XX (Ubelaker, 2003). Estos datos sugieren que las lesiones debidas a enfermedades infecciosas, desorden del crecimiento, y los niveles de infección están aumentando durante el Formativo Temprano pero todavía no alcanzan los valores más altos de los periodos posteriores. La hiperostosis porótica todavía no aparece en el Formativo Temprano, lo que sugiere según este investigador que la subsistencia y la densidad de población no han creado condiciones propicias para la anemia grave en las poblaciones. Sin embargo se encuentran evidencias de hiperostosis porótica en la costa en el Periodo Precontacto Intermediario en las poblaciones Guangala (Verano \& Ubelaker, 1992) y luego manteñas (Delabarde, 2006; 2010; Graber \& Jastremski, 2009).

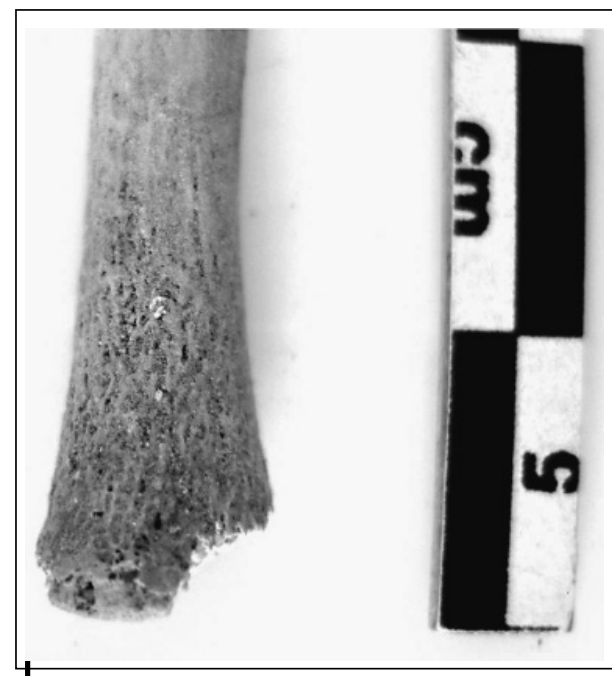

Figura 3 - Lesiones de periostitis en la superficie de todos los huesos largos de un individuo adulto encontrado en una urna funeraria (tola J6, J6-III)

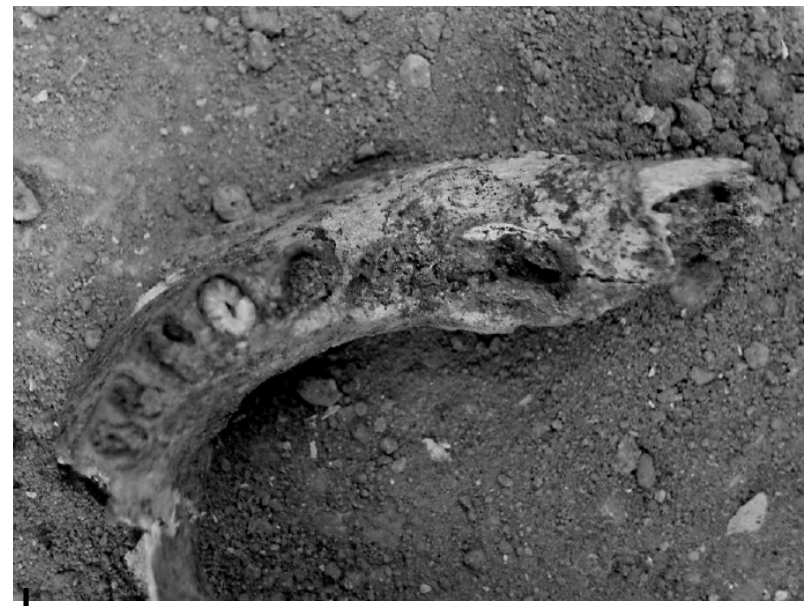

Figura 4 - Esta mandíbula de adulto (ind\#2) presenta un absceso dental por debajo del segundo molar superior derecho, con la pérdida ante mortem de este diente (Tola J5) 


\section{2. Primeros resultados del estudio de la dieta: el aporte del análisis de los isótopos estables}

Las patologías más comunes encontradas en la población de Japoto son entonces los desordenes nutricionales (síndromes carenciales como la anemia) y/o del desarrollo y las enfermedades infecciosas (Delabarde, 2010).

Paralelamente, se encontraron en Japoto numerosas «empanadillas» asociadas a las ocupaciones funerarias como domésticas. Las empanadillas, así denominadas en un primer momento por la investigadora Mercedes Guinea, eran tierras arcillosas modeladas de diversos tamaños y formas (tipo tamal 37 \% [fig. 5], tipo bollo $49 \%$ y tipo humita 4 \%) ligeramente cocinadas en hojas de vegetales (Guinea, 2006). El análisis de su composición mineralógica bajo microscopio óptico y electrónico de barrido y la técnica de difracción de rayos $X$ revelaron una mezcla de arcillas calcáreas diferentes con el predominio de la calcita, con un contenido importante de hierro y otros minerales. Su composición sugirió un posible consumo como suplementos dietéticos y/o tratamientos naturales para las enfermedades infecciosas. La práctica de la geofagia fue relatada en algunos libros de cronistas o viajeros para varias poblaciones de América del Sur, de la Amazonia hacia la Sierra (Guinea, 2006). Otros ejemplos de estas empanadillas fueron encontrados en el sitio arqueológico de Atacames en la costa norte del Ecuador ocupado desde el Formativo hacia el Periodo Histórico con vestigios importantes de la cultura manteña (Guinea, 2006).

La relación entre las lesiones osteológicas y el posible uso de tierras comestibles por parte de la población manteña de Japoto se planteó como una posible hipótesis de investigación, decidiéndose la realización de un estudio de los isótopos estables para establecer el consumo de las mismas.

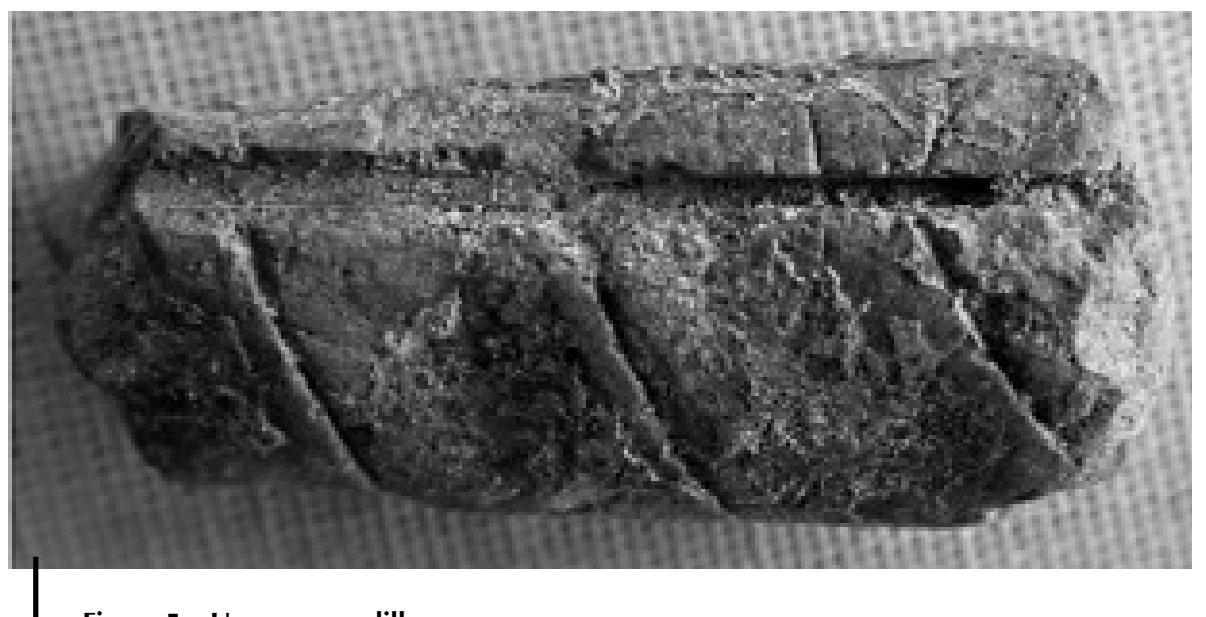

Figura 5 - Una empanadilla

Foto: proyecto Japoto 
En 2009, hemos mandado 7 muestras (6 huesos y 1 empanadilla) a Estelle Herrscher del laboratorio de la unidad de Antropología Biocultural en la Universidad de Marsella (Francia). Los resultados de este estudio preliminar provienen en gran parte de su informe (Herrscher, 2010).

A partir del análisis de isótopos estables de nitrógeno y carbono contenidos en la fracción proteica del hueso (colágeno), es posible evaluar el comportamiento alimentario de las poblaciones del pasado. Por un lado, los niveles isotópicos del nitrógeno $\left(\delta^{15} \mathrm{~N}\right)$ y el carbono $\left(\delta^{13} \mathrm{C}\right)$, medidos en los huesos corresponden a los acumulados durante la vida del individuo. Por otro lado, estos niveles se correlacionan con la composición de la dieta.

El análisis de las firmas isotópicas del nitrógeno $\left(\delta^{15} \mathrm{~N}\right)$ es de particular interés para restituir los diferentes niveles tróficos de una cadena alimenticia. El colágeno de los predadores presenta valores isotópicos superiores en nitrógeno a los del colágeno de sus presas. Así, cuando los sujetos ocupan los últimos eslabones de una cadena alimentaria (alto nivel trófico), sus firmas isotópicas de nitrógeno son elevadas. En cuanto al análisis de las firmas isotópicas del carbono $\left(\delta^{13} \mathrm{C}\right)$, este identifica la fuente alimenticia de carbono y en consecuencia, el tipo de medio ambiente en el que el hombre dispone de plantas. Un análisis más fiable y pertinente implica un estudio de las características isotópicas del ecosistema de la población estudiada. Sin embargo, como en el caso de Japoto, se pueden utilizar datos isotópicos publicados (Ortner, 1992; Barberena et al., 2009).

Las muestras óseas de Japoto $(\mathrm{N}=6)$ provenían de 6 individuos adultos (3 individuos masculinos, un individuo femenino y dos indeterminados) de tres tolas distintas $(\mathrm{J} 6, \mathrm{~J} 7$ y J8). Fue posible extraer colágeno con un rendimiento superior de 47,6mg.g $\mathrm{g}^{-1}$ de 5 muestras. Solo una de las muestras dio menos de 1 mg de colágeno (individuo femenino), estando también demasiado degradado para hacer un análisis isotópico. Los análisis previos de extracción revelaron una conservación homogénea de colágeno humano de las muestras de Japoto. Las 5 muestras presentaron una relación $(\mathrm{C} / \mathrm{N})$ dentro de los límites establecidos para el colágeno inalterado y sin modificación.

Los resultados del análisis isotópico de las muestras óseas revelan valores del carbono entre -9,2 y -8,5\% grados. Los valores isotópicos del nitrógeno entre el 12,9 y el 13,6 \%o. Los 5 individuos comparten similitudes en los valores isotópicos del nitrógeno-15 o del carbono-13. Dicho resultado refleja un régimen alimenticio isotópicamente similar para estas cinco personas. Para los sujetos de Japoto, los valores del carbono $\delta^{13} \mathrm{C}$ son muy altos indicando no solo el posible aporte de las empanadillas, sino también de plantas C4, y/o contribución de los animales de carne de forrajeo con las plantas C4 (probablemente el maíz). Los altos valores de nitrógeno $\left(\delta^{15} \mathrm{~N}\right)$, a su vez indican el consumo de alimentos enriquecidos en nitrógeno, como la carne y/o los peces marinos.

La empanadilla también fue analizada para establecer sus firmas isotópicas ya que no existía ningún estudio sobre el consumo de tierra comestible. Se emplearon dos preparaciones distintas de las muestras, la primera de la tierra misma y la segunda después de un lavado al acido para quitar todos los minerales de la tierra. 
La investigadora Estelle Herrscher consideró más adecuado medir los valores directamente en la tierra componente de la empanadilla; así las firmas isotópicas medidas corresponderían a las de los alimentos directamente consumidos.

Las muestras analizadas directamente muestran un porcentaje muy bajo de nitrógeno $(0,03$ y 0,06$)$. Este resultado es consistente con una fuente de proteínas muy baja en este alimento. Los valores isotópicos de carbono muestran valores de $-10,2$ y $-10,9 \%$ y los valores de nitrógeno son 7,4 y 7,3\%. Estos datos comparados con los obtenidos en otros contextos arqueológicos de América del Sur muestran similitudes con los valores isotópicos esperados para las plantas C4 como el maíz. La coincidencia entre los valores isotópicos de las empanadillas y las plantas C4 hace difícil determinar o distinguir la contribución de cada uno de estos alimentos en la dieta de la población manteña.

En conclusión, los individuos de Japoto presentan valores isotópicos superiores a las poblaciones consumidores de peces y de maíz del periodo poscontacto en Florida (Larsen et al., 2001). Por el contrario, para los valores del nitrógeno, se observa que los individuos de Japoto se encuentran en la variabilidad de criterios de las poblaciones poscontacto de Florida.

Una comparación similar realizada con los individuos de la cultura de PuruchucoHuaquerones del Perú muestra que los sujetos de Japoto tienen valores más altos de carbono y nitrógeno. Aunque estas comparaciones son empíricas debido a la falta de un análisis isotópico del medio ambiente, los sujetos de Japoto parecen haber consumido recursos alimenticios isotópicamente más enriquecidos en nitrógeno-15 y carbono-13 que las empanadillas. Este estudio preliminar sobre la dieta de la población manteña de Japoto, permite, dentro de los límites ya mencionados, destacar el consumo de alimentos enriquecidos en carbono-13 (el aporte de las empanadillas es posible pero no se puede excluir que el aporte se deba a plantas como el maíz), los valores altos del nitrógeno que reflejan el consumo de alimentos enriquecidos en nitrógeno-15 (peces, invertrebrados marinos, mamíferos terrestres, pájaros) y finalmente el aporte de animales terrestres consumidores de maíz. Este estudio preliminar constituye la primera fase de la investigación sobre la dieta de la población manteña; la segunda fase está enfocada en el estudio isotópico del medio ambiente a partir de los restos óseos animales encontrados asociados a las estructuras domésticas en Japoto y en el incremento de muestras óseas humanas para aumentar la fiabilidad y pertinencia de las conclusiones.

\section{3. Traumatismos: las evidencias osteológicas}

La mayoría de las lesiones encontradas en los esqueletos prehispánicos se dividen en tres categorías de enfermedades generales: infecciones, artritis y traumatismos. La patología más común son los cambios degenerativos. Los traumatismos ocupan el segundo lugar y afectan el esqueleto de varias maneras: fractura, dislocación o deformación de los huesos, lesiones de los tejidos blandos, venas y nervios en la periferia (White, 2000). 
Cuando el tejido óseo está vivo y en remodelación continua, las lesiones traumáticas se denominan ante mortem cuando existen evidencias de reacción ósea, peri mortem cuando la lesión no presenta reacción ósea pero tiene las características propias de haber sido infligida sobre un tejido óseo «fresco», y finalmente post mortem cuando las lesiones están asociadas a fenómenos tafonómicos afectando un hueso «seco».

En la población de Japoto, no tenemos muchos ejemplos de traumatismos óseos ante mortem o peri mortem. Hasta la fecha, solo se ha observado un ejemplo de un traumatismo ante mortem: el peroné izquierdo de un adulto (ind \# 2) proveniente de un depósito secundario (Z3) de la tola $\mathrm{J} 7$ presenta una fractura diafisaria desplazada del peroné izquierdo en su extremo proximal. El individuo ha sobrevivido un tiempo suficiente para permitir el desarrollo del cal óseo alrededor de la fractura (fig. 6).

Generalmente la gran frecuencia de lesiones traumáticas en las poblaciones arqueológicas es asociada a épocas de violencia. Ubelaker (2003: 284) por ejemplo, menciona en su estudio de los esqueletos del sitio de Real Alto la existencia, en el Periodo Formativo Temprano en el Ecuador, de un conflicto entre

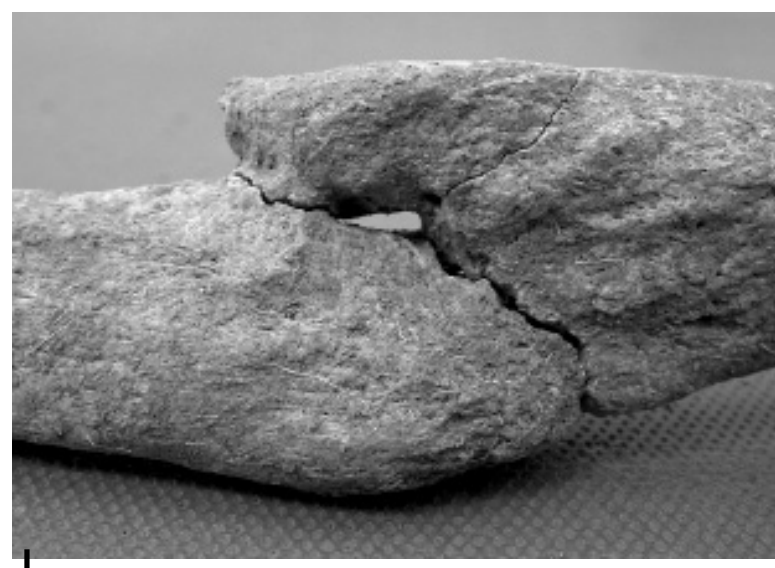

Figura 6 - Fractura diafisaria desplazada del peroné izquierdo en su extremo proximal de un adulto (ind \# 2) (Tola J7, Z3) grupos para el control de un territorio y de sus recursos, pero sin excluir los problemas de violencia social en la población misma.

No se registran evidencias de deformación craneana en Japoto, sin embargo esta práctica está presente en el sitio costeño de la Tolita (provincia de Esmeraldas) en los cráneos de 4 individuos de los periodos Clásico y Tardío (200BC400AD) (Ubelaker, 1997).

Se registró un solo ejemplo de traumatismo peri mortem en los esqueletos estudiados de Japoto. Se trataba de una bóveda craneana humana encontrada, en la esquina NE de la Estructura 1 de la tola J8, en la base del talud reforzado de la plataforma (ver Guinea este mismo volumen). La bóveda se encontró incompleta con el hueso frontal bastante completo y ambos parietales representados por algunos fragmentos. El borde supra-orbital izquierdo era muy pronunciado lo que era consistente con un individuo de sexo masculino. Sin embargo, había que notar que un solo carácter del dimorfismo sexual no permitía establecer la estimación del sexo. El aspecto general de la bóveda y el estado de fusión de las suturas craneales presentes permitieron decir que se trataba de un individuo adulto sin poder precisar la edad (fig. 7).

Esta bóveda craneana presenta varias evidencias muy características de la acción tafonómica (fracturas, desprendimiento del cortex y aspecto blanquecido). En los 
traumatismos post mortem es más fácil distinguir si estos se deben a acciones tafonómicas o se han producido en el momento de la excavación, como puede ser el impacto de la pala del arqueólogo.

Sin embargo, una fractura presente en la bóveda craneana muestra características distintas a las lesiones post mortem. Se localiza en la porción derecha del frontal a $45 \mathrm{~mm}$ del vertex y $57 \mathrm{~mm}$ del borde supra-orbital derecho partiendo del hueso parietal y corriendo hacia el frontal en dirección de la órbita derecha. Los bordes de las fracturas observados macroscópicamente y microscópicamente son muy nítidos, implicando que el tejido óseo está hidratado para que la fractura tenga este aspecto gracias a las características plásticas del hueso «fresco» (fig. 8). Los bordes de la fractura no presentan las características resultantes en caso de exposición al fuego. En cambio, el aspecto de esta fractura es compatible con un impacto con un objeto contundente, con la energía disipándose a través de la dicha fractura. Estas conclusiones constituyen una hipótesis ya que la bóveda craneana está bastante incompleta y no se puede excluir la presencia de otro tipo de lesión en las partes ausentes.

El descubrimiento de una bóveda craneana con fracturas peri mortem producidas por un posible impacto con un objeto contundente en la base de la plataforma de la Estructura 1 de la tola J8 recuerda la existencia en algunas culturas prehispánicas de sacrificios o muertes rituales asociados a la construcción o renovación de los edificios importantes (Bourget, 1998; Delabarde \& Uceda, 2003; Hocquenghem, 2005; Chávez Balderas, 2005). Mercedes Guinea (ver este mismo volumen) ha planteado la hipótesis del entierro ritual de la estructura citada.

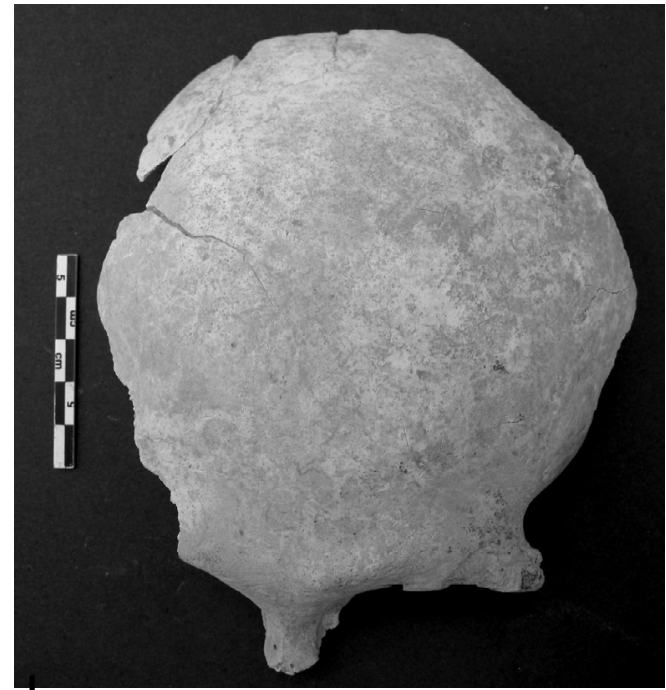

Figura 7 - Bóveda craneana encontrada en la base de la plataforma de la Estructura 1 de la tola J8

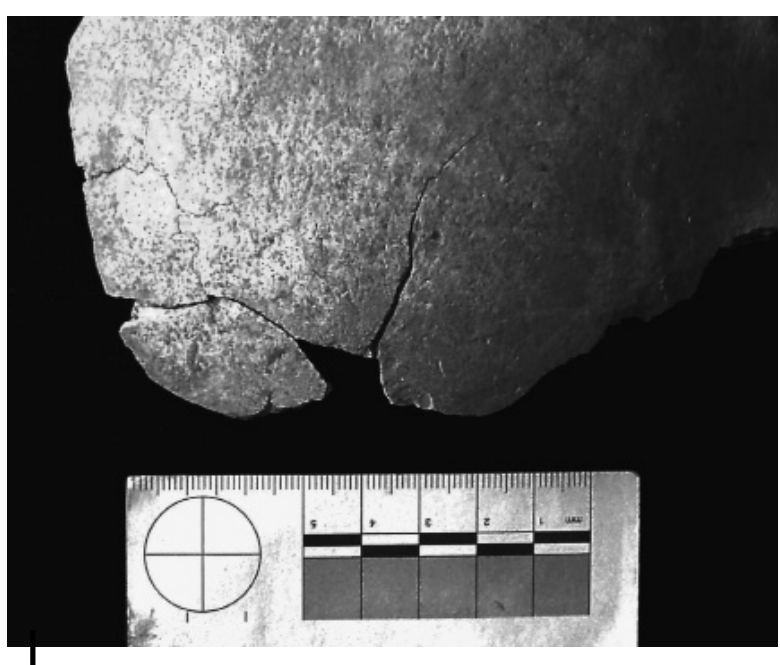

Figura 8 - Detalles de la fractura de la bóveda craneana (Tola J8) 
A pesar de los límites de nuestro muestreo, la poca frecuencia de traumatismos óseos en Japoto es concordante con los otros estudios antropológicos (Ubelaker 1981; 1997; Graber \& Jastremski, 2009) con un índice más bajo de lesiones traumáticas en el Periodo Precontacto y un índice más alto en los Periodo Formativo y Poscontacto (Ubelaker, 2003).

\section{CONCLUSIÓN}

La interrelación entre la biología y la cultura es una problemática dinámica y compleja. Los restos humanos ofrecen datos biológicos (estado sanitario, dieta) y culturales (prácticas funerarias) que deben ser estudiados. Primero dentro del contexto mismo del sitio con el análisis del material arqueológico asociado y luego en comparación con los demás datos de poblaciones vecinas cronológicamente y geográficamente.

El estudio antropológico de los restos humanos encontrados en Japoto $(\mathrm{N}=39$ individuos) permite tener una idea de la vida y la muerte de una población manteña, tomando en consideración los límites del muestreo debido a las prácticas funerarias (selección de los individuos, representación esquelética incompleta en los depósitos secundarios) y la deficiente conservación de los restos óseos.

Las lesiones encontradas más frecuentemente están relacionadas con los cambios degenerativos, ya sean por enfermedad reumática o por actividades específicas (transporte de carga). Existen evidencias de enfermedades infecciosas activas en el momento de la muerte, algunas lesiones relacionadas con la anemia o malnutrición y escasas lesiones traumáticas. Las enfermedades dentales, frecuentes en los consumidores de maíz, deben haber tenido mayor importancia pero se recuperaron pocos dientes en nuestro muestreo. No se ha podido extraer ADN en cantidad y calidad suficiente para obtener datos interpretables. En cambio, el colágeno de los huesos humanos está bastante bien preservado como para permitir un análisis de isótopos estables. Se ha podido establecer el consumo de alimentos enriquecidos en carbono-13 (empanadillas y/o plantas como el maíz) y en nitrógeno-15 (peces, invertebrados marinos, mamíferos terrestres) y animales terrestres consumidores de maíz. Estos datos son coherentes con el material arqueológico encontrado (empanadillas, huesos animales y pescados, piedras de moler para el maíz).

Sin caer la imagen idílica del Nuevo Mundo exento enfermedad antes de la Conquista, el estudio antropológico de la población manteña de Japoto muestra un estado sanitario satisfactorio dentro de los parámetros antropológicos de poblaciones contemporáneas. El Periodo de Integración en el Ecuador es conocido como una época relativamente estable. El acceso a recursos marítimos y terrestres reflejados a través de la dieta variada, el posible uso de tierras comestibles como remedios a algunas enfermedades infecciosas, y la construcción de numerosas tolas ilustra en Japoto una población dominando y beneficiándose de su medio ambiente. 


\section{Referencias citadas}

AUSTIN ALCHON, S., 1999 - Las grandes causas de la muerte en la América Precolombina. Una perspectiva hemisférica. Papeles de Población, 21: 199-221.

BARBERENA, R., GIL, A. F., NEME, G. A. \& TYKOT, R. H., 2009 - Stable Isotopes and Archaeology in Southern South America. Hunter-Gatherers, Pastoralism and Agriculture: An Introduction. International Journal of Osteoarchaeology, 19: 127129.

BOURGET, S., 1998 - Pratiques sacrificielles et funéraires au site Moche de la Huaca de la Luna, côte nord du Pérou. Bulletin de I'Institut Français d'Études Andines, 27 (1): 41-74.

BROOKS, S. T. \& SUCHEY, J. M., 1990 - Skeletal age determination based on the os pubis: A comparison of the Ascáadi-Nemeskeri and Suchey-Brooks methods. Human Evolution, 5: 227-238.

BUIKSTRA, J., 1992 - Diet and Disease in Late Prehistory. In: Disease and Demography in the Americas (J. Verano \& D. H. Ubelaker, eds.): 87-101; Washington, D.C.: Smithsonian Institution Press.

CHÁVEZ BALDERAS, X., 2005 - Sacrificio humano y tratamientos mortuorios en el Templo Mayor de Tenochtitlán. Informe Presentado a FAMSI.

CIEZA DE LEÓN, P., 1984 [1553] - Obras completas, 3 vols.; Madrid: Consejo Superior de Investigaciones científicas.

DENEVAN, W., COOK, S. F. \& BORAH, W., 1973 - Essays in Population History: Mexico and the Caribbean, Vol. 1, xxiii + 455 pp.; Berkeley, California: University of California Press. Journal of Latin American Studies, 5: 289-290. doi:10.1017/ S0022216X00007562

DELABARDE, T., 2006 - Una secuencia de patrones funerarios manteños en la provincia de Manabí: primeros resultados de la tola 7, sitio de Japoto (Ecuador). Bulletin de I'Institut Français d'Études Andines, 35 (3): 313-320.

DELABARDE, T., 2010 - From one burial to another: a sequence of funerary patterns from the Manteña culture in the province of Manabi, site of Japoto (Ecuador). In: Return of the Living Dead: Funerary Practices and Models in the Ancient Andes (P. Eeckout \& L. Stewart Owens, eds.); Cambridge: Cambridge University Press.

DELABARDE, T. \& UCEDA, S., 2003 - Les Sacrifices Rituels des Mochicas : analyse des traces d'instruments tranchants sur les os humains. Pour la Science, 303: 80-84.

DIOMEDI, A. P., 2003 - La guerra biológica en la conquista del nuevo mundo. Una revisión histórica y sistemática de la literatura. Revista Chilena de Infectiologia, 20 (1): 19-25.

GENOVÉS, S. C., 1967 - Proportionality of Long Bones and Their Relation to Stature Among Mesoamericans. American Journal of Physical Anthropology, 26: 67-78.

GRABER, Y. \& JASTREMSKI, N., 2009 -Étude d'une tombe collective de l'époque Manteño (Salango, Équateur) dans son contexte, culturel et funéraire, régional. Antropo, 18: 9-25. www.didac.ehu.es/antropo

GUINEA, M., 2006 - El uso de tierras comestibles por los pueblos costeros del Periodo de Integración en los Andes septentrionales. Bulletin de I'Institut Français d'Études Andines, 35 (3): 321-334.

HERRSCHER, E., 2010 - Culture précolombienne en Équateur: Analyses isotopiques de quelques sujets humains. Rapport d'étude manuscrit. 
Japoto, Ecuador: salud, enfermedad y muerte. Evidencias osteológicas y dentales

HOCQUENGHEM, A. M., 2005 - Sacrificios y calendario ceremonial en las sociedades de los Andes centrales. In: Chamanismo y sacrificio: perspectivas arqueológicas y etnológicas en sociedades indígenas de América del Sur (J.-P. Chaumeil, R. Pineda Camacho \& J.-F. Bouchard, eds.): 75-104; Bogotá: IFEA, Banco de la República, Fundación de investigaciones arqueológicas nacionales.

ISCAN, M.Y., LOTH, S. R. \& WRIGHT, R. K., 1984 - Metamorphosis at the sternal rib end: a new method to estimate age at death in white males. American journal of physical anthropology, 65 (2):147-56.

ISCAN, M. Y., LOTH, S. R. \& WRIGHT, R. K. 1985 - Age estimation from the rib by phase analysis: white females. Journal of forensic sciences, 30 (3): 853-863.

JAFFE, A. J. 1992 - The first Immigrants from Asia, 358 pp.; New York: Plenum Press.

LARSEN, C. S., GRIFFIN, M. C., HUTCHINSON, D. L., NOBLE, V. E., NORR, L., et al., 2001 - Frontiers of Contact: Bioarchaeology of Spanish Florida. Journal of World Prehistory, 15: 69-123.

ORTNER D. J. 1992 - Skeletal pathology: Probabilities, Possibilities and Impossibilities. In: Disease and Demography in the Americas (J. Verano \& D. H. Ubelaker, eds.): 5-13; Washington, D.C.: Smithsonian Institution Press.

ORTNER, D. J. 2003 - Identification of pathological conditions in human skeletal remains; London: Elseiver Academic Press. Segunda edición.

SCHEUER, L. \& BLACK, S., 2000 - Developmental Juvenile Osteology, 587 pp.; USA: Academic Press Inc.

STECKEL, R. H. \& ROSE, J. C., 2002 - The Backbone of History: health and nutrition in the Western Hemisphere; New York: Cambridge University Press.

STOREY, R., 1992 - Life and Death in the Ancient City of Teotihuacan. A moderne paleodemographic synthesis, xvii + 306 pp.; Tuscaloosa: University of Alabama Press.

UBELAKER, D. H., 1981 -The Ayalán Cemetery: A Late Integration Period Burial Site on the south coast of Ecuador, 175 pp.; Washington D. C.: Smithsonian Institution. Smithsonian Contributions to Anthropology, 29.

UBELAKER, D. H. 1995 - Status and diet in Precontact Highland Ecuador. American Journal of Physical Anthropology, 97: 403-411.

UBELAKER, D. H., 1997 - Skeletal Biology of Humans Remains From la Tolita, Esmeraldas Province, Ecuador; Washington D. C.: Smithsonian Institution Press.

UBELAKER, D. H., 2003 - Health Issues in the Early Formative of Ecuador: Skeletal Biology of Real Alto. In: Archaeology of Formative Ecuador (J. Scott Raymond \& Richard L. Burger, eds.): 259-287; Washington, D.C.: Dumbarton Oaks Research Library and Collection.

VERANO, J. \& LOMBARDI, G., 1999 - Paleopatología en Sudamérica Andina. Bulletin de I'Institut Français d'Études Andines, 28 (2): 91-121.

VERANO, J. W. \& UBELAKER, D. H. (eds.), 1992 - Disease and Demography in the Americas; Washington, D. C.: Smithsonian Institution Press.

WALPER, U., CRUBEZY, E. \& SCHULTZ, M., 2004 - Is Cribra orbitalia synonymous with anemia? Analysis and interpretation of cranial pathology in Sudan. American Journal of Physical Anthropology, 123: 333-339.

WALKER, P. L., RHONDA, R. B., RICHMAN, R., GJERDRUM, T. \& ANDRUSHKO, V.A., 2009 - The causes of porotic hyperostosis and cribra orbitalia: A reappraisal of the iron-deficiency-anemia hypothesis. American Journal of Physical Anthropology, 139-2: 109-125. 
WELLS, C., 1965 - Bones, bodies and disease: Evidence of Disease \& Abnormalities. in Early Man: Ancient, 288 pp; London: Editions Praeger.

WHITE, T. D., 2000 - Human Osteology, 563 pp.; San Diego California: Academic Press. Segunda edición. 